\title{
NOTE
}

\section{Effects of Chlorpromazine and Estradiol Benzoate on Prolactin Secretion in Gonadectomized Male and Female Rats}

\author{
Sumio TAKAHASHI ${ }^{1}$ SEIICHIRo KAWASHIMA ${ }^{1}$ \\ AND KATSUMI WAKABAYASHI ${ }^{2}$ \\ ${ }^{1}$ Zoological Institute, Faculty of Science, University of Tokyo, \\ Tokyo 113, Japan and 'Institute of Endocrinology, \\ Gunma University, Maebashi, 371, Japan
}

\begin{abstract}
Synopsis
The effects of chlorpromazine (CPZ) and estradiol benzoate (EB) on serum prolactin (PRL) levels were studied in gonadectomized male and female rats. In both sexes CPZ $(25 \mathrm{mg} / \mathrm{kg}$ body weight) produced an elevation of PRL when measured $2 \mathrm{hr}$ after the injection, but the elevated levels were higher in ovariectomized rats than in orchidectomized rats. These results reconfirm a sexual difference in the regulatory mechanism of PRL secretion in response to the dopamine receptor blocker. Pretreatment with $5 \mu \mathrm{g}$ EB $48 \mathrm{hr}$ before $\mathrm{CPZ}$ injection abolished this sexual difference in serum PRL concentration.
\end{abstract}

Several investigators have suggested that there is a sexual difference in the elevation of serum prolactin (PRL) concentration after the blockage of the hypothalamic inhibition (Bishop et al., 1972; Krulich et al., 1975 ; Ojeda et al., 1977). It is claimed that dopamine (DA) is in itself a PRLinhibiting factor and directly acts at the pituitary level (Brown et at., 1976 ; Horowski and Gräf, 1976; Macleod, 1976). Ojeda et al. (1977) recently reported that serum PRL levels after the injection of pimozide, a DA receptor blocker, were higher in female rats than in male rats, and suggested that the sexual difference in PRL secretion was partly caused by the long-term effect of estrogen during the course of sexual development. Chlorpromazine (CPZ) is thought to be another DA receptor blocker (Horn and Snyder, 1971) and increased the basal serum PRL concentrations by the

Received September 21, 1978. blockage of dopaminergic inhibitory control (Lu et al., 1970). In the present study, the effect of CPZ with or without estrogen treatment on serum PRL concentrations was compared between orchidectomized and ovariectomized rats. Gonadectomy was performed in all groups of rats to remove the difference in the circulating sex steroid hormones.

\section{Materials and Methods}

Male and female rats of the Wistar strain were used in the present experiments. At 8 weeks of age 16 male and 20 female rats were gonadectomized under ether anesthesia. Two weeks after gonadectomy 5 males and 7 females received an intraperitoneal injection of CPZ (Contomin, Yoshitomi Pharmaceutical Ind. Ltd., Osaka) in a dose of 25 $\mathrm{mg} / \mathrm{kg}$ body weight (Groups B and E). Other 6 males and 6 females were given a subcutaneous injection of $5 \mu \mathrm{g}$ estradiol benzoate (EB, Sigma) in $0.2 \mathrm{~m} l$ sesame oil $48 \mathrm{hr}$ before CPZ treatment (Groups $\mathrm{C}$ and $\mathrm{E})$. The remaining 5 males and 7 females re- 
ceived an intraperitoneal injection of vehicle (consisting of $0.05 \%$ sodium pyrosulfite, $0.05 \%$ sodium sulfite and $\mathbf{0 . 0 7 5 \%}$ vitamin C) of $\mathrm{CPZ}$ only (Groups $A$ and D). Blood samples were collected under light ether anesthesia from the femoral vein before CPZ injection and from the carotid artery at autopsy $2 \mathrm{hr}$ after CPZ injection. At autopsy the anterior lobe of pituitary was quickly taken out, and weighed on a torsion balance to the nearest $0.05 \mathrm{mg}$. It was homogenized in $2 \mathrm{ml}$ of $0.05 \mathrm{M}$ phosphate buffer at $\mathrm{pH} 7.0$, frozen and thawed, then centrifuged at $3,000 \mathrm{rpm}$ for $12 \mathrm{~min}$. Serum samples and the supernatants of the homogenate were kept frozen at $-20^{\circ} \mathrm{C}$ until assay for PRL. PRL levels were determined with NIAMDD radioimmunoassay kit from Rat Pituitary Hormone Distribution Program, NIAMDD, National Institutes of Health, Bethesda, Md, according to the procedure described in the instruction paper with a slight modification. The assay results were expressed as mIU of NIAMDDrat prolactin-RP-1. The PRL levels between groups were analyzed by Student's t test.

\section{Results}

The serum PRL concentrations are presented in Fig. 1. In orchidectomized male rats there was some elevation in the level of serum PRL by CPZ injection, but the difference from the basal level before CPZ injection was statistically not significant $(0.05<\mathrm{P}<0.1$, Fig. 1 , Group B). In ovariectomized female rats the serum PRL level was significantly increased by $\mathrm{CPZ}$ injection $(\mathbf{P}<0.001$, Fig. 1, Group E). The serum PRL level of orchidectomized male rats $2 \mathrm{hr}$ after $\mathrm{CPZ}$ injection was significantly lower than that of ovariectomized female rats $(\mathrm{P}<0.02$, Fig. 1, Group B vs $\mathrm{E})$. There was no significant difference in the basal serum PRL levels between orchidectomized males and ovariectomized females before CPZ or vehicle injection.

In EB-pretreated gonadectomized rats the basal serum PRL levels were significantly higher than those in gonadectomized rats without EB pretreatment (Fig. 1, Group A vs $\mathrm{C}$, Group $\mathrm{D}$ vs $\mathrm{F}$ ). There was no difference in the basal serum PRL levels between EB-pretreated gonadectomized male and female rats (Fig. 1, Group $\mathrm{C}$ vs $\mathrm{F}$ ). In EB-pretreated orchidectomized males the serum PRL level significantly increased by $\mathrm{CPZ}$ injection $(\mathrm{P}<0.001$, Fig. 1, Group $\mathrm{C})$. Likewise, in ovariectomized females the elevated level of serum PRL by EB pretreatment was further increased by $\mathrm{CPZ}$ injection $(\mathrm{p}<0.05$, Fig. 1, Group F).

Fig. 2 demonstrates the total PRL contents (mIU/gland) or concentrations (mIU/ $\mathrm{mg}$ ) of the anterior pituitary $2 \mathrm{hr}$ after CPZ or vehicle injection. The pituitary PRL contents or concentrations did not show any significant changes by CPZ injection or EB plus CPZ injection, compared with those in the controls in both sexes.

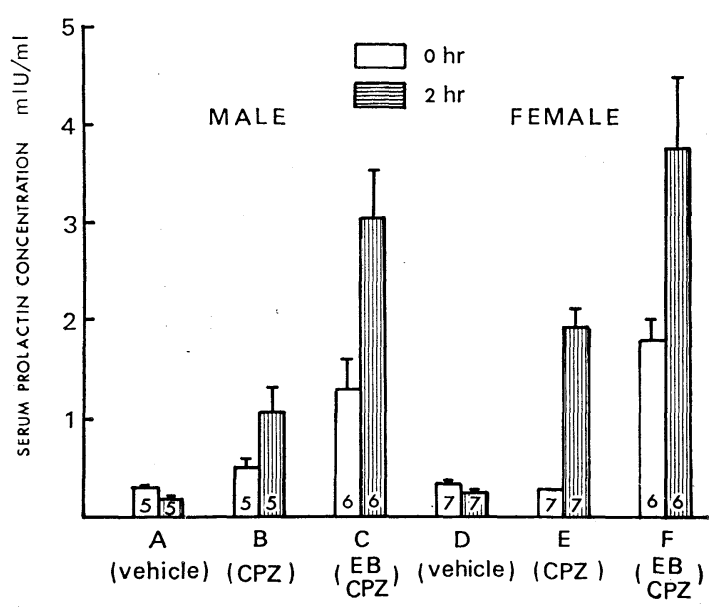

Fig. 1. Effects of CPZ on serum PRL concentrations in gonadectomized male and female rats with or without prior EB treatment. A=orchidectomized male rats without $\mathrm{EB}$ and without CPZ; $\mathrm{B}=$ orchidectomized male rats without $\mathrm{EB}$ but with $\mathrm{CPZ} ; \mathrm{C}=$ orchidectomized male rats with both EB and CPZ; D=ovariectomized female rats without $\mathrm{EB}$ and without $\mathrm{CPZ} ; \mathrm{E}=$ ovariectomized female rats without EB but with $\mathrm{CPZ} ; \mathrm{F}=$ ovariectomized female rats with both EB and CPZ. The open columns indicate the serum PRL concentrations before $\mathrm{CPZ}$ injection and the shaded columns indicate the serum PRL concentrations $2 \mathrm{hr}$ after the injection of CPZ (B, C, E and F) or vehicle of CPZ (A and D). Vertical lines depict standard error of the means, and the number in each column indicates the number of rats used. 

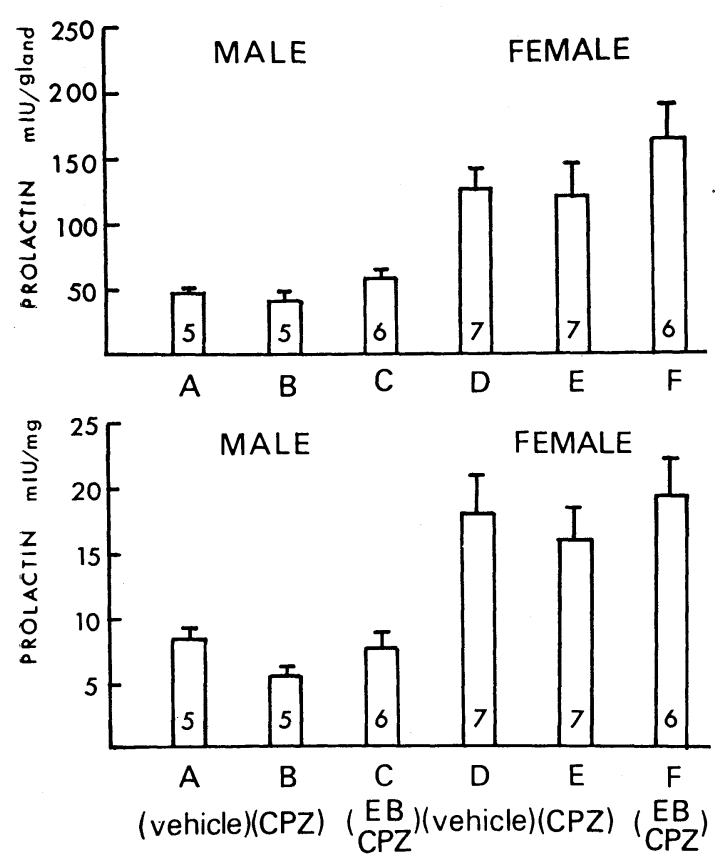

Fig. 2. Comparison of the pituitary PRL contents (mIU/gland) and concentrations (mIU/mg) $2 \mathrm{hr}$ after CPZ treatment or vehicle injection in gonadectomized male and female rats with or without EB treatment. For the abbreviation of Group A-F, see the legends in Fig. 1.

In female rat pituitaries PRL contents and concentrations were decidedly more than those in male rat pituitaries, regardless of prior treatments.

\section{Discussion}

The present study showed a sexual difference in the elevation of serum PRL concentrations by $\mathrm{CPZ}$ in gonadectomized rats. This finding is in agreement with those of Ojeda et al. (1977), who recently reported that in gonadectomized rats as well as in intact rats serum PRL concentrations were higher in female rats than in male rats after the injection of pimozide.

The difference in the basal serum PRL concentrations between gonadectomized male and female rats was not observed. However, the pituitary PRL contents or concentrations were higher in ovariectomized females than in orchidectomized males. Therefore, as suggested by Ojeda et al. (1977), PRL release seems to be more strongly held in check in ovariectomized females than in orchidectomized males.

EB pretreatment tended to cause a more pronounced increase in the basal serum PRL concentrations in ovariectomized females than in orchidectomized males, but the difference was statistically not significant. In contrast, Mallampati and Johnson (1973) reported that the hypothalamo-pituitary axis in ovariectomized female rats was more responsive to a multiple injections of estrogen on PRL secretion than in orchidectomized male rats. In the present experiments, $\mathrm{CPZ}$ administration to EB-pretreated rats significantly increased the EB-pretreated levels of serum PRL in both sexes, but the significant sexual difference was not detected. The sexual difference in PRL secretion visualized by CPZ in gonadectomized rats was blunted by EB pretreatment.

\section{Acknowledgements}

The authors wish to express their gratitudes to Dr. A. F. Parlow and Rat Pituitary Hormone Program, NIAMDD, NIH for the kind supply of RIA kit. This investigation was supported in part by a Grant-in-Aid for Scientific Research (Nos. 154257 and 234047) from the Ministry of Education, Science and Culture, Japan.

\section{References}

Bishop, W., C. P. Fawcett, L. Krulich and S. M. McCann (1972). Endocrinology 91, 643.

Brown, G. M., P. Seeman and T. Lee (1976). ibid. 99, 1407.

Horn, A. S. and S. H. Snyder (1971). Proc. Nat. Acad. Sci. USA 68, 2325.

Horowski, R. and K.-J. Gräf (1976). Neuroendocrinology 22, 273. 
Krulich, L., E. Hefco and J. E. Aschenbrenner (1975). Endocrinology 96, 107.

Lu, K., Y. Amenomori, C. Chen and J. Meites (1970). ibid. 87, 667.

Macleod, R. M. Frontiers in Neuroendocrinology, Vol. 4 (edited by L. Martini and W. F. Ganong). Raven Press, New York, p. 169 (1976).

Mallampati, R. S. and D. C. Johnson (1973). J. Endocrinol. 59, 209.

Ojeda, S. R., A. Castro-Vázquez and H. E. Jameson (1977). Endocrinology 100, 427. 